\title{
Control based bifurcation analysis for experiments
}

\author{
By Jan Sieber, Bernd Krauskopf \\ Bristol Centre for Applied Nonlinear Mathematics, Department of Engineering \\ Mathematics, Queen's Building, University of Bristol, Bristol BS8 1TR, UK
}

\begin{abstract}
We introduce a method for tracking nonlinear oscillations and their bifurcations in nonlinear dynamical systems. Our method does not require a mathematical model of the dynamical system nor the ability to set its initial conditions. Instead it relies on feedback stabilizability, which makes the approach applicable in an experiment. This is demonstrated with a proof-of-concept computer experiment of the classical autonomous dry friction oscillator, where we use a fixed time step simulation and include noise to mimic experimental limitations. For this system we track in one parameter a family of unstable nonlinear oscillations that forms the boundary between the basins of attraction of a stable equilibrium and a stable stick-slip oscillation. Furthermore, we track in two parameters the curves of Hopf bifurcation and grazing-sliding bifurcation that form the boundary of the bistability region. An accompanying animation further visualizes the action of the controller during the tracking process.
\end{abstract}

Keywords: Bifurcation analysis, numerical continuation, hybrid experiments

\section{Introduction}

The simplest and most frequently encountered type of self-excited non-stationary behaviour in nonlinear dynamical systems are periodic oscillations. A common and typical scenario is a loss of stability of a stable equilibrium in a Hopf bifurcation when a complex conjugate pair of eigenvalues of the linearization crosses the imaginary axis under variation of a single parameter. At the critical parameter value a family of oscillations is born. If the system is nonlinear then the oscillations are nonlinear as well, and, close to the Hopf bifurcation, they are either stable (supercritical Hopf bifurcation) or unstable (subcritical Hopf bifurcation). This scenario is well understood theoretically and can be found in standard textbooks such as (Kuznetsov 2004). If one tracks the emerging family of periodic orbits (which correspond to the nonlinear oscillations) further, other bifurcations may be encountered. If the dynamical system is described mathematically in the form of a system of differential equations then there are numerical continuation tools available (Kuznetsov 2004, Doedel, et al. 1998) that can track families of periodic orbits regardless of their dynamical stability. What is more, these tools can also track curves of bifurcations in two-dimensional parameter planes. These curves form the boundaries of parameter regions with qualitatively different long-time behaviour of the dynamical system (for example, regions of stable steady states, stable nonlinear oscillations, 
or regions with more complicated behaviour such as quasiperiodic or chaotic motions). Thus, numerical continuation is a tremendously useful method if one knows the equations of motion and wants to understand the qualitative behaviour of a nonlinear system and how it depends on the system's parameters. For example, references (Kuznetsov 2004, Doedel et al. 1998) discuss and illustrate this approach with a long list of classical examples and provide an entry point into the extensive literature.

By contrast, if the dynamical system is given in the form of an experiment then the task of tracking periodic oscillations and their stability boundaries is quite challenging. One approach is to run the experiment close to its stability boundary for a sufficiently long time to determine if the transients decay or grow. However, the decay or growth of transients is typically very slow close to the stability boundary. Moreover, the polynomial effects caused by nonnormality (Trefethen \& Embree 2005) interfere for long periods of time if the exponential decay/growth is weak. Finally, small perturbations are amplified due to the lack of damping in the critical directions. Thus, the approach of observing transients is time-consuming and produces results of low accuracy.

In this paper we present an alternative method for the tracking of unstable periodic orbits and their bifurcations in experiments. The fundamental assumptions for our method are, first, that the system is feedback stabilizable (as explained in $\S 4$ ) and, second, that the feedback control input and the system parameters of interest can be varied automatically with a precision that corresponds to the accuracy of the desired results. The core algorithm is a continuation routine which provides an iterative computational method that prescribes a sequence of control inputs and parameter values. This sequence eventually converges toward a non-invasive control input. The computations have to be performed in parallel to running the experiments, but not in real time. Importantly, it is not necessary to set initial values of the internal state variables of the experiment explicitly (which would involve stopping and reinitializing the experiment). Moreover, the dynamical system always remains in a stable regime with a closed stabilizing feedback loop, so that our method does not require to run the experiment freely close to its stability boundary.

The work presented here follows on from (Sieber \& Krauskopf 2006) where we introduced a control-based continuation scheme as an embedding of extended timedelayed feedback control (see also $\S 2$ ) and demonstrated its use for the continuation of periodic orbits of a dynamical system where only the output of a simulation is available. Here we extend this work to the continuation of periodic orbits and their bifurcations. Furthermore, we use projection onto Fourier modes to obtain a sufficiently robust continuation method that can be used even in the presence of limited precision and noise in the experimental measurement.

The performance of our method is demonstrated in a proof of concept with a classical mechanical system, namely the dry-friction oscillator, which we run as a computer experiment. To mimic the restrictions faced by experimenters we disturb the numerical simulation and its output by a small amount of noise and restrict the output to a discrete time series of fixed sampling stepsize. Specifically, we continue a family of unstable nonlinear oscillations in one parameter and curves of Hopf bifurcation and grazing-sliding bifurcation in two parameters. The action of 
the controller during the continuation is discussed and further visualized with an accompanying animation.

Our method is ideally suited for computer-controlled experiments, especially for hybrid tests such as real-time dynamic substructured testing of mechanical engineering systems (Blakeborough, et al. 2001, Kyrychko, et al. 2005). These tests couple an experimental test specimen of a poorly understood or critical component in real time (and bidirectionally) to a computer simulation of the remainder of the structure. One of the central goals of hybrid experiments is the tracking of stability boundaries. The automatic and precise variation of parameters and the feedback stabilizability, which our method requires, are particularly easy to achieve in hybrid tests (since they are in part run as a computer simulation).

The outline of the paper is as follows. We briefly discuss existing approaches for finding unknown periodic orbits and bifurcations in dynamical systems without model in $\S 2$. Section 3 introduces the dry friction oscillator, the example used throughout the paper. In $\S 4$ we give the details of the general control-based continuation algorithm for periodic orbits, and show in $\S 5$ how this procedure works for the family of unstable periodic orbits in the dry friction oscillator. In $\S 6$ we extend the core continuation to enable it to track Hopf and grazing-sliding bifurcations directly, and we demonstrate the tracking of bifurcations in the dry friction oscillator in $\S 7$. We conclude and outline further work in $\S 8$.

\section{Background on related methods}

One approach for finding periodic orbits and equilibria in dynamical systems without knowledge of equations of motion is stabilization with extended time-delayed feedback control (ETDFC). This method aims to stabilize unstable periodic orbits without prior knowledge of their time profile (Pyragas 1992, Pyragas 2001, Gauthier, et al. 1994). Its essential idea is to feed back a difference between the current state and the state from one or more periods ago, which nessecitates prior knowledge of the period of the orbit. In the case of equilibria the underlying idea can be further simplified to using adaptive (or washout) filters (Hassouneh \& Abed 2004). The main advantage of this method is that its implementation is possible for any experiment with feedback stabilizable periodic orbits and equilibria. Furthermore, the method avoids running the free dynamical system close to its stability boundary. However, it is unclear under which conditions the extended time-delayed or washout filtered feedback converges. Feedback stabilizability of the periodic orbit is necessary but not sufficient. The approach in (Sieber \& Krauskopf 2006) is to embed ETDFC into a continuation scheme, which allows for the continuation of periodic orbits in more general, but still limited situations, for example, through fold (saddle-node) bifurcations. As will become clear in $\S 4$, the method described in this paper has been inspired directly by the (continuation embedding of) ETDFC, but modifies it substantially in order to make it more robust. This additional robustness comes at the price of an increased computational effort.

A second method, known as equation-free coarse-grained (or time-stepper based) bifurcation analysis, is well-established in the context of microscopic simulations such as kinetic Monte Carlo simulations; see (Kevrekidis, et al. 2004) for a survey and references. This approach assumes that a small number of macroscopic quantities (such as the first few moments) already satisfy a closed system of ordinary 


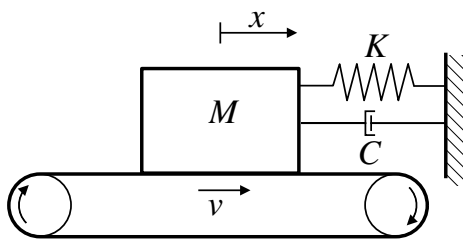

Figure 1. Set-up of an idealised dry friction oscillator.

differential equations (ODEs). The right-hand-side of this ODE and its partial derivatives are then evaluated by running appropriately initialized short bursts of a microscopic simulator. This procedure relies fundamentally on the ability to initialize the microscopic simulator 'at will', and on the implementation of a 'lifting operator' that maps the values of the macroscopic quantities to initial values of the ensembles of microscopic particles. If these two ingredients are given then a number of high-level tasks can be performed on the macroscopic level, including bifurcation analysis, optimization, controller design and control (Siettos, et al. 2004). Coarsegrained bifurcation analysis has been demonstrated successfully in the analysis of equilibrium dynamics of multi-particle systems. Since the microscopic simulator is treated as a 'computer experiment' the approach could, in principle, be applied to real experiments. The limiting factor in the adoption of this time-stepper based approach is the impossibility (or impracticality) to initialize the real experiment at will. In fact, initializing the system is particularly difficult if one aims to find non-equilibrium dynamics such as periodic orbits. In the continuation approach of our method the previous solution is used as seed in the Quasi-Newton step, so that the need for initializing the system does not arise.

Finally, both ETDFC and the method presented here rely on the existence of a successful feedback control. There exist standard techniques in control engineering that aim to identify the right-hand-side of the system locally by probing systematically various control inputs. The resulting linear identified system can be used subsequently to obtain bifurcation diagrams. (De Feo \& Maggio 2003) have demonstrated this for an implementation of the Colpitts oscillator as an electronic circuit. Their method assumes that the internal state of the dynamical system can be measured. However, in general, system identification is an inverse problem and, as such, inherently ill-posed. The method presented here, as well as ETDFC and coarse-grained bifurcation analysis, avoid the need for solving an inverse problem.

\section{The dry friction oscillator with constant forcing}

As our illustrating example we consider a dry friction oscillator with constant forcing as sketched in figure 1 . The friction between the running belt and the mass induces a force on the mass, pushing it against the damped spring that is fixed to the wall. The overall force on the mass at position $x$ is

$$
-K x-C \dot{x}-F_{f}(\dot{x}-v)
$$

where $x$ is measured with respect to the reference position of the relaxed spring, $K$ is the spring constant, $C$ is the damping, $F_{f}$ is the force exerted by the friction, and $v$ is the velocity of the running belt. Thus, the dynamics of this nonlinear single- 


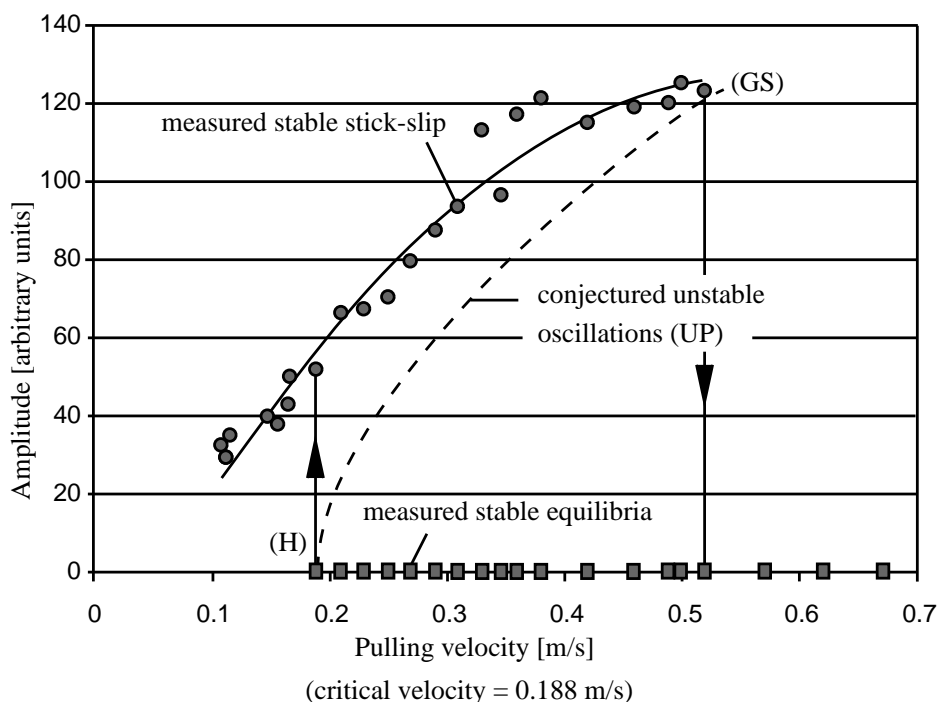

Figure 2. Diagram of the experimental results from (Horváth 2000) showing the velocity $v$ versus the amplitude of oscillations. Dots show the amplitude of measured stick-slip oscillations (fitted with a solid line) and squares refer to measured equilibria. The dashed line connecting the transitions is the conjectured family (UP) of dynamically unstable periodic orbits. (Reprinted with kind permission from G. Stépán; translations from the Hungarian original courtesy of G. Orosz.)

mass-spring-damper system is governed by an equation of motion of the form

$$
M \ddot{x}+C \dot{x}+F_{f}(\dot{x}-v)+K x=0 .
$$

It has been observed experimentally by (Horváth 2000) that the dynamics of the dry friction oscillator changes qualitatively when one varies the system parameter $v$. The set-up of (Horváth 2000) used a mass fixed to a bending beam on a large rotating disc to prevent disturbances by lateral degrees of freedom; see also (Stépán \& Insperger 2004). Figure 2 shows the sequence of experimental measurements from (Horváth 2000) for different values of the velocity $v$. The figure shows that for large $v$ the rest state (equilibrium) $x_{0}$, given by $x_{0}=-F_{f}(-v) / K$ and $\dot{x}=0$, is stable. At a critical velocity $v_{h} \approx 0.188$ the rest state $x_{0}$ loses its stability. If one decreases $v$ gradually from above $v_{h}$ to below $v_{h}$ one observes a sudden transition to large-amplitude stick-slip oscillations. Similarly, when increasing $v$ in the stick-slip oscillation regime the system jumps to a stable equilibrium at a critical velocity $v_{d}>v_{h}$. Figure 2 clearly shows this bistability in the parameter region $\left[v_{h}, v_{d}\right] \approx[0.188,0.52]$.

If one makes assumptions about all parameters in (3.2) the model can be analysed numerically using standard numerical methods such as AUTO (Doedel et al. 1998) and SlideCont (Dercole \& Kuznetsov 2005). For example, a friction model that supports the presence of the smooth linear instability at $v_{h}$ is

$$
F_{f}(w)=\left[F_{c}+\left(F_{s}-F_{c}\right) \exp (-|w|)+F_{v}|w|\right] \operatorname{sgn}(w)
$$

which gives the velocity-force curve shown in figure 3 . The argument $w$ is positive in (3.3) for non-sticking trajectories. The analysis of the numerical model (3.2) 


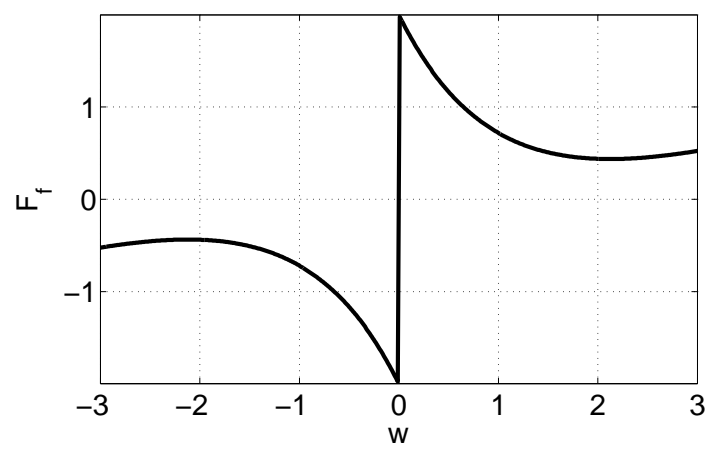

Figure 3. Friction law $F_{f}(w)$ used in (3.3) for the parameters $F_{c}=-0.5, F_{s}=2$ and $F_{v}=0.3$.

completes the diagram in Figure 2 and predicts the following (Galvanetto \& Bishop 1999):

(H) the event of loss of stability of the equilibrium is a subcritical Hopf bifurcation,

(UP) there is a family of non-sticking unstable nonlinear oscillations separating the two stable regimes for parameters $v$ in the interval $\left[v_{h}, v_{d}\right]$, and

(GS) the event where the stick-slip oscillations lose their stability is a grazing-sliding bifurcation, which is a non-smooth bifurcation; see (di Bernardo, et al. 1999) for a survey.

\section{Control-based continuation of periodic orbits}

We first explain the core idea behind control-based continuation of a family of periodic orbits such as the family (UP). The continuation of bifurcations is an extension of this core algorithm, which we explain in $\S 6$. For convenience of notation let us assume that the measured output $y(t) \in \mathbb{R}^{n}$ of the free-running experiment is governed by an ordinary differential equation (ODE), depending on a tunable parameter $\mu \in \mathbb{R}^{k}$ :

$$
\dot{y}=g(y, \mu)
$$

We denote a neighbourhood of a point or set $m$ by $U(m)$ and the space of continuous periodic functions on the unit interval by

$$
C_{p}\left([0,1] ; \mathbb{R}^{n}\right)=\left\{y \in C\left([0,1] ; \mathbb{R}^{n}\right): y(0)=y(1)\right\}
$$

Accordingly, $y \in C_{p}^{1}$ if $y$ is continuously differentiable and $\dot{y}(0)=\dot{y}(1)$. Let us assume that (4.1) has a smooth regular one-parameter family of periodic orbits

$$
\Gamma=\left\{\left(y_{s}, \mu_{s}, T_{s}\right) \in C_{p}^{1}\left([0,1] ; \mathbb{R}^{n}\right) \times \mathbb{R}^{k} \times \mathbb{R}: s \in \mathbb{R}\right\}
$$

That is, the number of parameters $k$ equals one. The periodic time profile $y_{s}$ satisfies $\dot{y}_{s}\left(T_{s} \cdot\right)=T_{s} g\left(y_{s}\left(T_{s} \cdot\right), \mu_{s}\right)$ where the argument of $y_{s}$ is the time scaled to the interval $[0,1], T_{s}$ is the period of the orbit, and $s \in \mathbb{R}$ is parametrizing the family $\Gamma$. 


\section{(a) Input-output map}

We assume that we can measure the time profile $y(t)$ and apply feedback control to the experiment such that it is governed by an ODE of the form

$$
\dot{y}=g(y, \mu)-h(y, \mu)[y(t)-\tilde{y}(t)] .
$$

In general we do not know $g$ and $h$. However, we assume that the control is able to achieve stabilization of (4.2). More precisely, we assume the existence of a smooth input-output map

$$
Y: U(\Gamma) \subset C_{p}\left([0,1] ; \mathbb{R}^{n}\right) \times \mathbb{R}^{k} \times \mathbb{R} \rightarrow C_{p}\left([0,1] ; \mathbb{R}^{n}\right)
$$

which is defined for $\left(y_{0}, \mu_{0}, T_{0}\right) \in U(\Gamma)$ in the following way. We set $\tilde{y}(t)=y_{0}\left(T_{0} t\right)$ as control target and $\mu=\mu_{0}$ in (4.2). Then (4.2) has a unique stable periodic orbit $y\left(T_{0} \cdot\right) \in C_{p}\left([0,1] ; \mathbb{R}^{n}\right)$ close to $y_{0}\left(T_{0} \cdot\right)$. The map $Y$ is defined by

$$
Y:\left(y_{0}, \mu_{0}, T_{0}\right) \in U(\Gamma) \mapsto y .
$$

Thus, the assumption of stabilization means that this periodic orbit $y$ exists, is (locally) unique for all $\left(y_{0}, \mu_{0}, T_{0}\right) \in U(\Gamma)$, and its dependence on $\left(y_{0}, \mu_{0}, T_{0}\right)$ is smooth. The period of $y$ is automatically $T_{0}$ and the linearization of $Y$ with respect to $y_{0}$ is compact if the output of the experiment is indeed governed by an ODE. A function $y$ is a periodic orbit of the free experiment governed by (4.1) if and only if it is a fixed point of the map $Y\left(\cdot, \mu_{0}, T_{0}\right)$. The same is true for higher iterates $Y^{m}$ of $Y$ if (4.2), when replacing $h$ by $m h$, still has a regular linearization in $y$. This implies that one can also consider fixed point problems of higher iterates of $Y$, which can improve the convergence of the (Quasi-)Newton iteration introduced in $\S 4(\mathrm{~d})$.

Each evaluation of the map $Y$ in some point $\left(y_{0}, \mu_{0}, T_{0}\right)$ involves setting the parameter to $\mu_{0}$, the control target to $y_{0}\left(T_{0} \cdot\right)$ and running the controlled experiment until it has reached a stable state $y$ of period $T_{0}$. This stable state $y$ is (at least locally) independent of the initial condition. The basic idea of our control-based continuation method is to embed the fixed point problem for $Y\left(\cdot, \mu_{0}, T_{0}\right)$ into a pseudo-arclength continuation of the family $\Gamma$, extending the fixed point problem with a phase condition and a pseudo-arclength condition determining $\mu_{0}$ and $T_{0}$; see also (Sieber \& Krauskopf 2006).

The evaluation of $Y$ is potentially time-consuming and (in real experiments) of low accuracy compared to numerical computations with double precision floating point arithmetic. This fact has to be taken into account in the algorithm for discretizing and solving the fixed point problem. However, this control-based approach has two advantages that make it particularly practical for application to experimental dynamical systems. First, in the context of a continuation it is sufficient that the control (4.2) works in the neighbourhood $U(\Gamma)$ as the transients occurring in the controlled experiment (4.2) (when evaluating $Y$ ) are typically small. Second, it is not necessary to set the initial conditions for the internal states between successive evaluations of $Y$. In the continuation procedure we only assume the existence and smoothness of $Y$ without referring to (4.1) or (4.2). Thus, the method works whenever feedback control input $u[\tilde{y}-y]$ based on some output $y$ is able to stabilize the observable part of a dynamical system. We avoid any explicit system identification of the right-hand-side, which is an inverse and, thus, inherently ill-posed problem. 


\section{(b) Discretization of the fixed point problem}

At the core of the continuation procedure is the parameter dependent nonlinear fixed point problem for $y$

$$
y=Y(y, \mu, T)
$$

with the variables $(y, \mu, T) \in U(\Gamma)$. The popular extended time-delayed feedback control of unstable periodic orbits (Pyragas 1992, Gauthier et al. 1994) is equivalent to the approach of solving the fixed point problem (4.4) with the relaxed simple fixed point iteration $y_{l+1}=(1-\kappa) y_{l}+\kappa Y\left(y_{l}, \mu, T\right)$ for $l=1,2 \ldots,|\kappa|<1$, fixed $\mu$, and the 'correct' $T$.

We choose the more robust (and more expensive) alternative of a Quasi-Newton iteration where the infinite-dimensional variable $y$ is discretized with a Galerkin projection. A Quasi-Newton iteration requires an estimate of the linearization of $Y$ which can often only be evaluated with a low precision. Thus, the Galerkin basis elements must have a large support compared to the basic sampling interval (scaled by $T$ ) of the output profile $y$ of (4.2) such that the projection has an averaging effect, which increases the accuracy. This is in contrast to most common numerical discretization schemes that can afford to choose basis elements with a small support to obtain sparse matrices (Trefethen 1996). For our demonstrations in $\S 5$ and $\S 7$ we choose a Fourier mode basis whose elements have global support. For periodic orbits a projection

$$
\Phi: C_{p}\left([0,1] ; \mathbb{R}^{n}\right) \mapsto \mathbb{R}^{(2 q+1) \times n}
$$

onto the first $q$ Fourier modes is defined by

$$
\Phi[y]_{l}=\int_{0}^{T} b_{l}(t / T) y(t) d t \quad \text { where } b_{l}(t)= \begin{cases}\sqrt{2 / T} \cos (2 \pi l t) & \text { for } l=-q \ldots-1 \\ \sqrt{1 / T} & \text { for } l=0, \\ \sqrt{2 / T} \sin (2 \pi l t) & \text { for } l=1 \ldots q .\end{cases}
$$

This projection gives rise to a discretized version of the fixed point problem (4.4) for $\varphi \in \mathbb{R}^{(2 q+1) \times n}$ consisting of the $2 q+1$ variables $\varphi_{l} \in \mathbb{R}^{n}$ :

$$
\varphi_{l}=\Phi_{l}\left[Y\left(\sum_{j=-q}^{q} \varphi_{j} b_{j}, \mu, T\right)\right],
$$

which we write in compact form as

$$
\varphi=Y_{q}(\varphi, \mu, T) \text { where } Y_{q}: \mathbb{R}^{(2 q+1) \times n} \times \mathbb{R}^{k} \times \mathbb{R} \mapsto \mathbb{R}^{(2 q+1) \times n} .
$$

One evaluation of $Y_{q}$ requires one evaluation of $Y$ and, thus, running the controlled experiment (4.2) once. Moreover, for each evaluation of $Y_{q}$ the variable $\varphi$ has to be converted to its scaled time profile $y(t)=\sum_{l=-q}^{q} \varphi_{l} b_{l}(t)$ and the result has to be converted back using the projection $\Phi$. In practical applications, where time profiles are sampled on a discrete time mesh, this conversion can be done efficiently using the Fast Fourier Transform and its inverse.

\section{(c) Embedding into a pseudo-arclength continuation}

We embed the discretized fixed point problem (4.7) into a continuation by including the parameter $\mu \in \mathbb{R}$ as a variable and appending two equations to obtain a 
regular system of $(2 q+1) n+2$ equations for the variable $\eta=(\varphi, \mu, T) \in \mathbb{R}^{(2 q+1) n+2}$. Let us assume that $j \geq 1$ points $\eta_{j}$ of $\Gamma$ have been computed already and that $\eta_{t, j}$ is an approximation for the tangent to $\Gamma$ in the last point $\eta_{j}$ (for example, the secant $\eta_{t, j}=\left(\eta_{j}-\eta_{j-1}\right) /\left\|\eta_{j}-\eta_{j-1}\right\|$ if $\left.j>1\right)$. In a classical predictor-corrector pseudo-arclength continuation the next point $\eta=(\varphi, \mu, T)$ of $\Gamma$ has to satisfy

$$
\varphi=Y_{q}(\eta)
$$

extended by the pseudo-arclength condition

$$
\left[\eta_{t, j}\right]^{T}\left[\eta-\eta_{p}\right]=0
$$

where $\eta_{p}=\eta_{j}+s \eta_{t, j}$ is a prediction that can also be used as a starting point of the corrector iteration. The stepsize $s$ along $\Gamma$ is small and determines the approximate distance between the last point $\eta_{j}$ and the new point $\eta$ on the branch. For oneparameter families of generic periodic orbits the dimension $k$ of the parameter $\mu$ equals one. In this case $\eta$ is completely determined by (4.8) and (4.9) plus a phase condition such as

$$
\sum_{l=-q}^{q} l\left[\varphi_{l}\right]^{T} \varphi_{j,-l}=0
$$

which guarantees that the projected (and scaled) time profiles $y(t)=\sum_{l=-q}^{q} \varphi_{l} b_{l}(t)$ satisfy $\int_{0}^{1} y(t)^{T} \dot{y}_{j}(t) d t=0$. Condition (4.10) fixes the phase of the periodic orbit in a way that avoids large transients (and, possibly, failure of the control) during the evaluation of $Y$. This condition is also implemented, for example, in AUTO (Doedel et al. 1998) to optimize the mesh adaptation.

\section{(d) Linearization and solution of the nonlinear corrector system}

The correction step of the continuation is the iterative solution of the nonlinear system (4.8)-(4.10). The main costs within each iteration arise from the cost of obtaining the right-hand-side and its linearization with respect to the variable $\eta$ and the subsequent solution of the linear system of equations. If the dimension $n$ of $y$ is moderate (say, $n \leq 10$ ) and the time profiles $y(t)$ are moderately non-harmonic (which implies that we can choose the number of Fourier modes $q$ moderate, say, $q<$ 100) then the cost of obtaining the linearization far outweighs the cost of the linear algebra because the linearization $\partial_{\eta} Y_{q}$ of $Y_{q}$ is a dense matrix that can, in general, only be obtained by a finite-difference approximation. Thus, obtaining the complete finite-difference approximation of $\partial_{\eta} Y_{q}$ requires in the worst case $(2 q+1) n+k+1$ runs of the controlled experiment (4.2). The matrix $\partial_{\varphi} Y_{q}$ is dense independently of the choice of the Galerkin basis. In fact, the linearization of the original input-output map $Y$ with respect to its argument $y_{0}$ in (4.3) is dense, that is, $Y\left(y_{0}, \mu_{0}, T_{0}\right)(t)$ in general depends on $y_{0}\left(t^{\prime}\right)$ for all $t^{\prime} \in[0,1]$.

Near the Hopf bifurcation the matrix $\partial_{\varphi} Y_{q}$ is approximately blockdiagonal with respect to the Fourier modes (that is, $\partial\left[\left(Y_{q}\right)_{l}\right] / \partial \varphi_{m} \approx 0$ if $|m| \neq|l|$ ) because the coefficients of the linearization of (4.2) do not depend on time in the Hopf point. Thus, near the Hopf bifurcation the linearization of $Y_{q}$ can be obtained by taking $2 n+k+1$ finite differences.

For these reasons, (damped) Quasi-Newton iterations, for example, based on Broyden's update formulas (see (Eyert 1996) for a recent comparative study on 
update schemes), are more attractive than in the classical continuation schemes implemented and discussed by (Doedel et al. 1998, Kuznetsov 2004). The continuation of periodic orbits in the example in $\S 5$ starts at the Hopf bifurcation. Thus, we can start the Quasi-Newton iteration with a fully initialized blockdiagonal Jacobian matrix that we update with Broyden updates of rank $\nu \leq 2$ along the curve. We update the Jacobian directly instead of its inverse because in this way we can incorporate the analytically known derivatives of extending equations such as (4.9) and (4.10). Thus, during the corrector iteration we update the old estimate $A_{\text {old }}$ for $\partial_{\varphi} Y_{q}$ to the new estimate $A_{\text {new }}$ satisfying

$$
A_{\text {new }} \Delta=R \text { and } A_{\text {new }} \Delta^{\perp}=A_{\text {old }} \Delta^{\perp}
$$

where $\Delta=\left[\eta^{\nu}-\eta^{0}, \ldots, \eta^{1}-\eta^{0}\right]$ is a list of differences of recent arguments and $R=\left[Y\left(\eta^{\nu}\right)-Y\left(\eta^{0}\right), \ldots, Y\left(\eta^{1}\right)-Y\left(\eta^{0}\right)\right]$ is the corresponding list of residuals.

\section{Tracking periodic orbits of the dry friction oscillator}

This section demonstrates how the continuation introduced in $\S 4$ can be used to find the family $\Gamma$ of unstable nonlinear oscillations (UP), which has been conjectured to exist in the dry-friction oscillator in $\S 3$, and its dependence on the parameter $v$.

(a) Set-up of the simulation of the controlled experiment

The assumption of feedback stabilization means that we are able to apply a feedback force to the mass of the form

$$
f\left[x-\tilde{x}_{1}, \dot{x}-\tilde{x}_{2}\right]=-\left(k_{1}\left[x-\tilde{x}_{1}\right]+k_{2}\left[\dot{x}-\tilde{x}_{2}\right]\right) .
$$

One option to achieve this (in approximation) is a feedback adjustment of the position of the wall if $C$ is small.

Our demonstration is based on a computer simulation of the model (3.2) with feedback

$$
M \ddot{x}+C \dot{x}+F_{f}(\dot{x}-v)+K x=-\left(k_{1}\left[x-\tilde{x}_{1}\right]+k_{2}\left[\dot{x}-\tilde{x}_{2}\right]\right)
$$

with the parameters $F_{c}=-0.5, F_{s}=2$ and $F_{v}=0.3$ in the friction law (3.3) and with the dimensionless parameters $K=1, M=1, k_{1}=1, k_{2}=2$, and $C=0$.

In order to mimic some of the restrictions of an experimental situation we add uniform noise of modulus 0.01 to the right-hand-side of (5.2) and to the output, and restrict the evaluation of the time profile $x(t)$ to a sampling in constant steps of length 0.01 . Throughout $\S 5$ and $\S 7$ we treat the numerical simulation of $(5.2)$ as an experiment and refer also to it as such (even though it is performed on the computer).

Each Fourier mode $\varphi_{l}$ of the control target is in $\mathbb{R}^{2}$, consisting of a position component (corresponding to $\tilde{x}_{1}$ ) and a velocity component (corresponding to $\tilde{x}_{2}$ ). The parameter $\mu \in \mathbb{R}^{1}$ is the belt velocity $v$. 
(b) Initialization and method parameters

We have chosen the following method parameters. In (4.8) we replace $Y_{q}$ by its second iterate. That is, we solve

$$
\begin{aligned}
& 0=\varphi-Y_{q}\left(Y_{q}(\varphi, \mu, T), \mu, T\right) \\
& 0=\sum_{l=-q}^{q} l\left[\varphi_{l}\right]^{T} \varphi_{j,-l} \\
& 0=\left[\eta_{t, j}\right]^{T}\left[\eta-\eta_{p}\right]
\end{aligned}
$$

for $\eta=(\varphi, \mu, T)$ instead of (4.8)-(4.10), to achieve a more rapid decay of contributing Fourier modes and, thus, a more robust convergence of the damped QuasiNewton iteration. The number of modes $q$ equals 10. For a given control target profile $y_{0}$, parameter $\mu_{0}$, and period $T_{0}$ we accept the output $y$ as stabilized in $t$ if it satisfies the $\mathbb{L}^{2}$-norm condition

$$
\frac{1}{T_{0}}\left[\int_{-T_{0}}^{0}\left\|y(t+s)-y\left(t+s-T_{0}\right)\right\|^{2} d s\right]^{1 / 2}<\varepsilon
$$

(computed by a sum over all sampled steps in the period $T_{0}$ ) where $\varepsilon=10^{-3}$. We can choose the tolerance $\varepsilon$ smaller than the noise level because the integral in (5.4) averages over all sampling steps $(\approx 600$ per period). Hence, the accuracy of evaluations of $Y_{q}$ is $\approx 10^{-3}$ (since $q \ll 600$ ).

We begin the continuation of the family $\Gamma$ at the Hopf bifurcation, initializing the Jacobian of the determining nonlinear system by a block-diagonal finitedifference approximation. The Fourier components $\varphi_{l}$ of the initial periodic orbit $\eta_{1}$ are approximately zero for all $l \geq 2$. The detection of the Hopf bifurcation and the iteration toward the initial periodic orbit $\eta_{1}$ approximating the Hopf bifurcation are discussed in more detail in $\S 7$. An approximate initial tangent vector along $\Gamma$ is the first Fourier mode $\varphi_{ \pm 1}$ of $\eta_{1}$. The tolerance for the convergence of the damped Quasi-Newton iteration is $5 \cdot 10^{-3}$ along $\Gamma$. We perform at least two iterations and accept the result if the $\mathbb{L}^{2}$-norms of the last proposed correction step and the residual $\delta$ of the right-hand-side of (5.3) are below tolerance. The damping factor is constant (namely 0.3 ) along $\Gamma$. Significantly sharper convergence tolerances are not sensible due to the restricted accuracy of the experimental evaluations of $Y_{q}$. The maximal (and initial) stepsize $s$ along $\Gamma$ is 0.05 .

\section{(c) Experimental effort during the continuation}

Figure 4 shows the result of the continuation of the family $\Gamma$. The continuation stops (the Quasi-Newton iteration fails to converge) at the grazing event when the minimal modulus of the relative velocity of the periodic orbit becomes zero. The initial orbit of the family (approximating the Hopf bifurcation) is marked by a circle in figure 4 . The orbit marked by a square approximately grazes; it has a maximal relative velocity $\max \tilde{x}_{2}-v \approx-0.05$. The two-parameter continuations in $\S 7$ start from these two marked orbits.

Figure 5 shows the temporal behaviour of the iteration along the part of $\Gamma$ that is highlighted by the grey rectangle in figure 4 . The motion of the mass on 


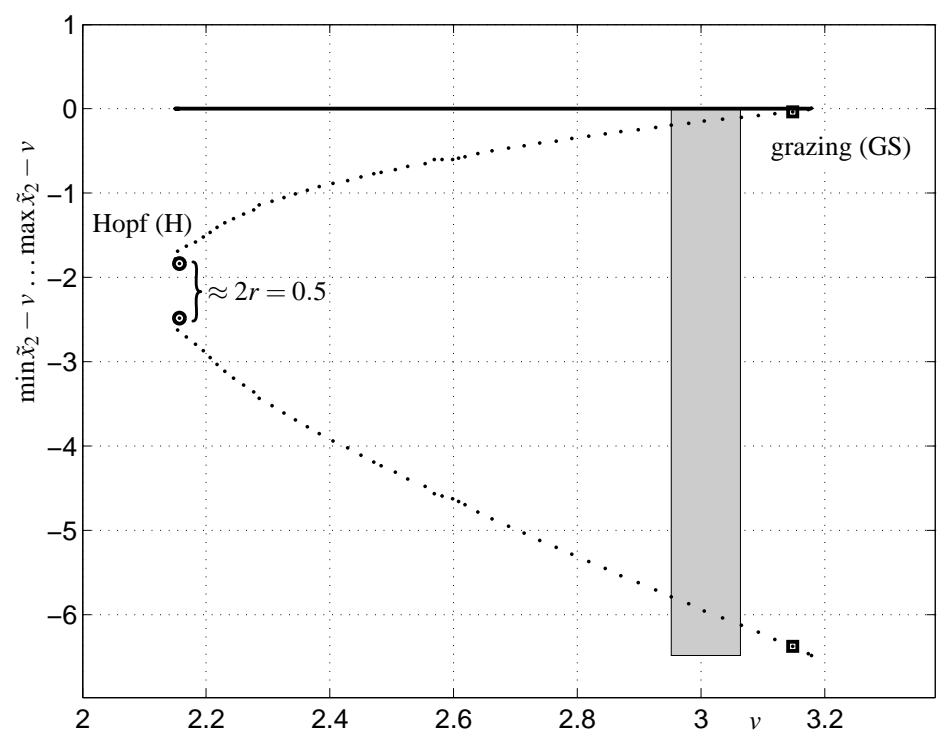

Figure 4. One-dimensional bifurcation diagram of family $\Gamma$ of periodic orbits. The dots show the minimal and maximal relative velocities $\tilde{x}_{2}-v$ of the periodic orbits $\left(\tilde{x}_{1}(t), \tilde{x}_{2}(t)\right)$ obtained by the continuation. The subsequent two parameter continuations started at the periodic orbits marked by a circle (H) and by a square (GS). The temporal behaviour of the continuation procedure within the grey rectangle is shown in detail in figure 5 .

the belt under the action of the controller during the continuation is shown in the accompanying animation. Panel (a) shows the time profile of the control target profile $\tilde{x}_{1}$. It does actually not change very much along this short segment of $\Gamma$, but notice the slight increase of the amplitude of the oscillation. Figure $5(\mathrm{~b})$ shows the position component $x-\tilde{x}_{1}$ of the control, and panel (c) shows the logarithm of the residual $\delta$ of the right-hand-side of the determining system (5.3). More precisely, the time coordinates of the crosses in panel (c) are the times $t$ at which the stabilization condition (5.4) is satisfied. At these times we evaluate the residual and change the arguments $\varphi, \mu$ and $T$. The comparison to the output $x$ in panel (a) shows that the relaxation toward an acceptable state takes often only two periods. The squares in panel (c) of figure 5 mark the starts of periods which also meet the convergence criterion of the Quasi-Newton iteration. Notice from figure 5(b) how the control target profile oscillates when a new step is started before settling down to zero. Figure 5(d) and (e) display the parameter $v$ and the period $T$, which are both automatically adjusted during the continuation process. The large steps are due to the predictor; the smaller changes occur during the Quasi-Newton iterations.

According to figure 5 the Quasi-Newton iteration needs on average only three to four evaluations of the right-hand-side to meet the convergence criterion. This makes it more efficient than a full Newton iteration based on Jacobian matrices obtained by finite-difference approximations. The continuation of the whole family $\Gamma$ to the last converging point needs 205 evaluations of the right-hand-side and takes 2740 dimensionless time units $(\approx 430$ periods). This excludes the initial full Newton iteration which took six iterations (thus, 42 evaluations of the right-hand-side). 


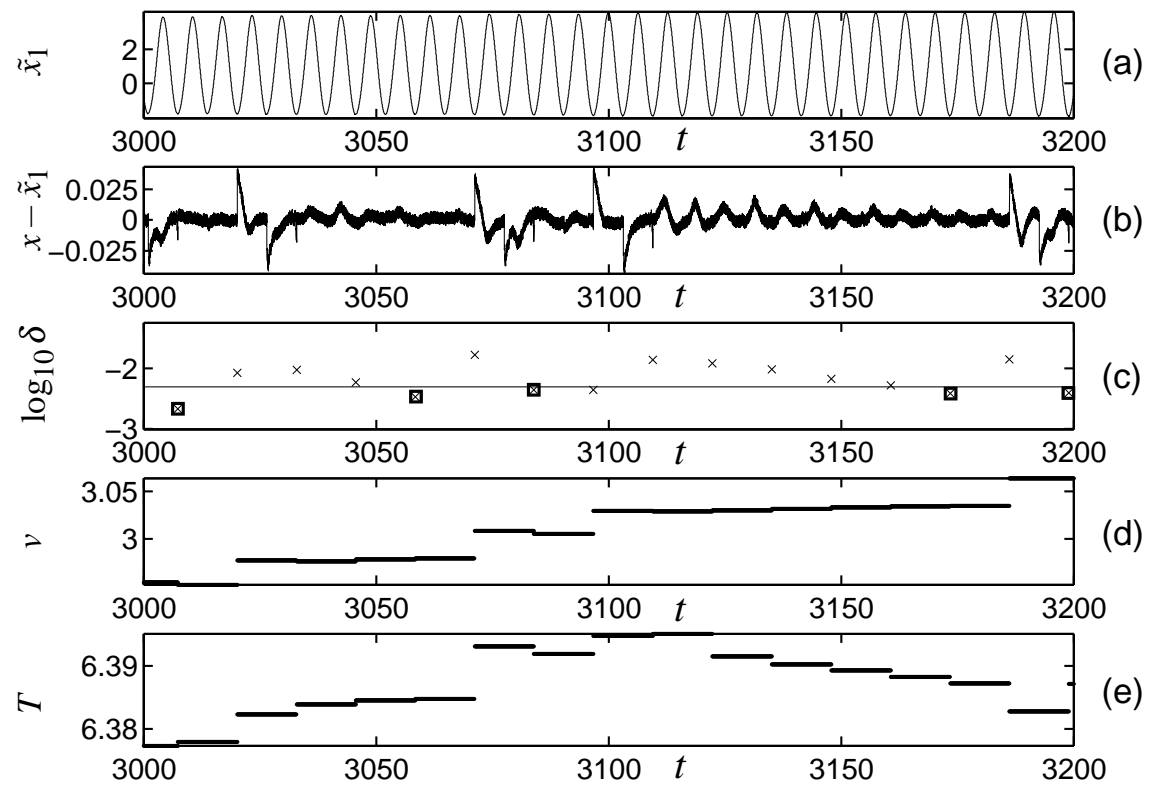

Figure 5. Time profiles along the part of $\Gamma$ in the grey region of Fig. 4. Panel (a) shows the control input $\tilde{x}_{1}$, panel (b) the difference $x-\tilde{x}_{1}$ between input and output, panel (c) the logarithm of the norm of the residual $\delta$ of the right-hand-side of system (5.3), and panels (d) and (e) show the bifurcation parameters $v$ and $T$. Crosses in panel (c) mark that the next period has been accepted as successful evaluation of the map $Y$, squares mark that the next period has been accepted as converged. See also the accompanying animation.

\section{Continuation of bifurcations}

A practical advantage of the continuation procedure introduced in $\S 4$ in the context of an experiment is that it can be extended quite easily to the direct tracking of bifurcations. The only additional requirement on the experimental set-up is the ability to vary two system parameters. Bifurcations are typically defined by additional conditions on the system variables. The continuation introduced in $\S 4$ operates only on variables that are easily accessible: the control target time profile $y_{0}$, a system parameter $\mu_{0}$ and the control period $T_{0}$. Thus, additional conditions on these variables can be introduced without any change in the experimental set-up. We demonstrate how this can be achieved for the two bifurcations that occur in the dry-friction oscillator from $\S 3$.

\section{(a) Continuation of a Hopf bifurcation}

The direct continuation of a Hopf bifurcation (such as in event $(\mathrm{H})$ in $\S 3$ ) can be approximated by continuing a periodic orbit of fixed small radius and harmonic time profile. We follow the continuation scheme (5.3) with the modification that we have $k=2$ (the dimension of the parameter $\mu$ ), choose the number of Fourier modes $q=1$ (since the motion is almost harmonic near the Hopf bifurcation), and extend system (5.3) by the equation

$$
\left[\varphi_{-1}\right]^{T} \varphi_{-1}+\left[\varphi_{1}\right]^{T} \varphi_{1}-r^{2}=0
$$


with a moderately small fixed radius $r$. The variable $\varphi=\left(\varphi_{-1}, \varphi_{0}, \varphi_{+1}\right)$ has dimension $3 n$, giving rise to an overall dimension $3 n+3$ for $\eta=(\varphi, \mu, T)$. Note that this size is considerably smaller than that required for the continuation of a general periodic orbit, which cannot be assumed to be harmonic and requires a much larger number of Fourier modes $q$. The component $\varphi_{0}$ is an approximation of the Hopf equilibrium and the components $\varphi_{-1} / r$ and $\varphi_{+1} / r$ approximate the pair of eigenvectors corresponding to the Hopf eigenvalue $2 \pi i / T$. Importantly, it is not necessary to evaluate $Y$ to obtain the bifurcation condition (6.1) and its linearization. Thus, in an experimental context the continuation of Hopf bifurcations does not require any additional experimental effort compared to the basic algorithm of $\S 4$.

We mention two possible difficulties of our approach of continuing Hopf bifurcation. First, due to the assumption that the periodic orbit of radius $r$ is close to harmonic, the Hopf bifurcation continuation may potentially generate spurious solutions. However, these can be detected (and then disregarded) by a large discrepancy between the full output time profile $y(t)$ of $(4.2)$ and its projection onto the first Fourier mode after each evaluation of $Y$. Second, tracking a family of small periodic orbits is problematic close to a Bogdanov-Takens bifurcation. At these generic end points of Hopf bifurcation curves in a two-parameter plane the pair of complex conjugate Hopf eigenvalues becomes a double zero eigenvalue and the period $T$ tends to infinity. This provides a possibility to detect the approach to a Bogdanov-Takens bifurcation. Note that we do not encounter Bogdanov-Takens bifurcations in this paper.

\section{(b) Continuation of the grazing event in the dry-friction oscillator}

If we rely on a bit of problem-specific a-priori knowledge we can even continue non-smooth bifurcations such as the grazing event (GS) in $\S 3$ approximately in two parameters. We know that the grazing orbits are exactly those that have a single point with zero relative velocity and that these orbits are unimodal close to their grazing points (that is, their phase portraits have no points of zero curvature). Thus, in the case of the dry-friction oscillator we can continue the grazing event (GS) approximately by extending the system (5.3) with the condition

$$
\max _{t \in[0,1]} \sum_{l=-q}^{q} \varphi_{l, 2} b_{l}(t)-v=d
$$

The value $d<0$ is the small maximal relative velocity of the periodic control target with the velocity profile $\tilde{x}_{2}(t)=\sum_{l=-q}^{q} \varphi_{l, 2} b_{l}(T t)$. The negative $d$ implies that we approximate the grazing event by continuing a smooth periodic orbit that 'almost' grazes. The unimodality of the periodic orbits near their minimal relative velocity guarantees that condition (6.2) is indeed differentiable with respect to $\varphi$. Again, condition (6.2) is not a condition on the experimental output but only on the control target and the parameter $v$. Thus, its approximate linearization can be obtained accurately by a computation of finite differences without additional experimental effort. 


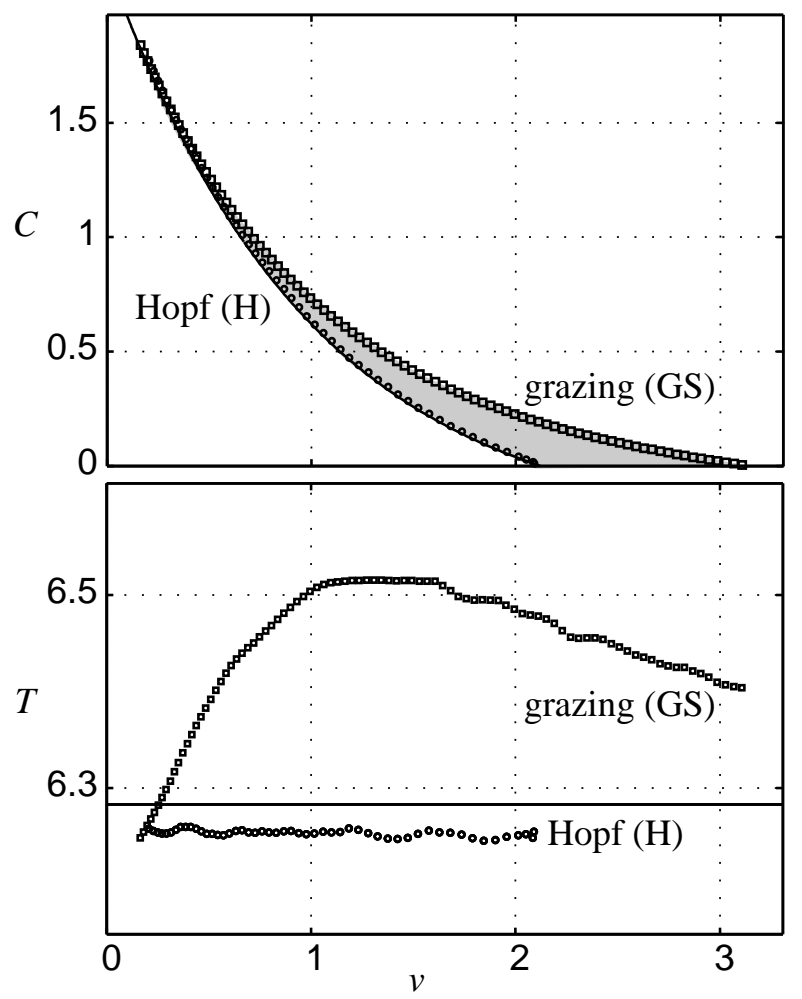

(a)

(b)

Figure 6. Tracked curves $\mathrm{H}$ (circles) and GS (squares) shown in the $(v, C)$-plane (a) and in projection onto the $(v, T)$-plane (b). The solid curve is the analytically known curve of Hopf bifurcation of (5.2). The region of bistability is shaded grey in panel (a)

\section{Bifurcation tracking for the dry friction oscillator}

Figure 6 shows the result of the two-parameter continuation of the Hopf bifurcation curve $\mathrm{H}$ and the grazing bifurcation curve GS in the critical velocity $\left(v_{h}\right.$ and $\left.v_{d}\right)$ and the viscous damping $C$. Note that the damping can be varied in an experiment, for example, by including a term of the form $C \dot{x}$ explicitly into the feedback control force $f$ in (5.1). Figure 6(a) shows the approximated curves in the $(v, C)$-plane and panel (b) shows the period of the respective periodic orbits as a function of their velocity $v$. The points representing $\mathrm{H}$ are marked by circles and those representing GS by squares.

The tracking of the curve $\mathrm{H}$ is started from the orbit with radius $r=0.25$; compare figure 4 . The value of $r=0.25$ was chosen to achieve a good trade-off between two counteracting effects. A large fixed $r$ approximates the Hopf bifurcation badly, whereas the condition of the Jacobian of system $(5.3),(6.1)$ is of order $O\left(r^{-1}\right)$, making a periodic orbit of very small radius difficult to track at low accuracies. The initial Hopf bifurcation point is determined by solving (5.3), (6.1) (a system of dimension nine) with a full Newton iteration for $j=0$ where we obtained our initial guess for $\varphi_{ \pm 1}$ from the transient behaviour of the free running system. (Robust general bifurcation detection is still an open problem without access to the righthand-side.) Only a crude approximation is necessary because almost every choice 
leads to a regular problem. The result of this iteration serves also as the initial periodic orbit of the continuation in $\S 5$.

In figure 6(a) the circles all lie (within the accuracy of the computation) on the analytically known Hopf bifurcation curve (solid curve) of (5.2). The period along the curve $\mathrm{H}$ is also close to the analytical value of $2 \pi$ indicated by the horizontal line in figure $6(\mathrm{~b})$. The tracking of the curve GS is started from the initial 'almost' grazing orbit with maximal relative velocity $\max \tilde{x}_{2}-v \approx-0.05$; compare figure 4 . Notice that the squares also appear to form a nice smooth curve in both figure $6(\mathrm{a})$ and (b), which is an indication of the accuracy of the computation.

As one would expect, the curves $\mathrm{H}$ and GS form the two boundaries of a region of bistability, which is shaded in figure 6(a). The approximation with non-zero $r$ in (6.1) and non-zero $d$ in (6.2) causes a systematic error H and GS, respectively. As a result, the size of the region of bistability is underestimated. (In our example, the systematic error has the side effect of adding a safety margin.) In particular, the curves $\mathrm{H}$ and GS already join (become tangent) for $v=v_{\min }>0$ and not only in the limit $v \rightarrow 0$. Note that the tracking of $\mathrm{H}$ becomes spurious as soon as the small periodic orbit that is tracked grazes, which happens at $v \approx r=0.25$. As was mentioned in $\S$ a this is detected and spurious solutions are disregarded.

\section{Conclusions and further work}

We have presented a method that allows for direct tracking of nonlinear oscillations and their bifurcations in nonlinear dynamical systems depending on two parameters. The method does not require a model of the dynamical system, so that it is applicable to the tracking of stability boundaries in experiments and substructuring tests. The feasibility of our approach in an experimental setting was demonstrated with the computer experiment of a friction oscillator. In the near future we are planning to implement our method in prototype substructured experiments, such as mass-spring-damper and mass-spring-pendulum systems (Kyrychko et al. 2005).

As presented here, our method uses Quasi-Newton iterations, which generally converge relatively slowly and generate full matrices. When problem specific information is available (apart from the choice of a suitable control method, which is simply assumed to be present in this paper) then the efficiency of the iteration can be increased substantially by incorporating known parts of the linearization into the Jacobian. For example, in mechanical systems often one half of the variables (the velocities) are time-derivatives of the other half of the variables, which reduces the dimension of the problem by half.

Our method can be extended (by formulating suitable conditions) to implement also the tracking of other bifurcations of equilibria and periodic orbits (also of periodically forced systems), such as period-doubling, saddle-node, and symmetrybreaking bifurcations. A more challenging task is the continuation of torus bifurcations and of strongly non-harmonic periodic orbits near homoclinic bifurcations. This requires other discretizations than projections onto Fourier modes, as well as a modification of the fixed point problem (4.8). Another interesting open problem is the efficient detection of bifurcations in an experimental setting; presently only saddle-node bifurcations are detected as folds of the continued branch (Sieber \& Krauskopf 2006). More generally, the goal is to implement the robust detection of stability changes directly in an experiment.

Article submitted to Royal Society 
An interesting topic of future research is the continuation of non-periodic trajectories and their stability changes. This capability would be extremely helpful in the context of substructured tests because it would enable one to decouple simulations and experiments dynamically. In effect, one would only need to match the interfaces at the end of each iteration. This approach would deal with two fundamental problems of the substructuring technology. First, coupling delays and the effects of unknown actuator dynamics would not play a role anymore and, second, the real-time restrictions on the computer simulation could be relaxed substantially.

\section{Acknowledgements}

The research of J.S. was supported by EPSRC grant GR/R72020/01, and that of B.K. by an EPSRC Advanced Research Fellowship.

\section{Electronic supplementary material}

The accompanying animation visualizes the time profiles as shown in figure 5 . The diagrams in the background show the bifurcation diagram, as it gradually completes during the continuation of the family of periodic orbits, and the time profile of the control. Black dots in the bifurcation diagram and black vertical lines in the control time profile correspond to acceptance of the control as non-invasive within tolerance. Red dots in the bifurcation diagram correspond to points where the right-hand-side of (5.3) has been evaluated.

\section{References}

A. Blakeborough, et al. (2001). 'The development of real-time substructure testing'. Philosophical Transactions of the Royal Society of London A 359:1869-1891.

O. De Feo \& G. Maggio (2003). 'Bifurcations in the Colpitts oscillator: from theory to practice'. Int. J. of Bifurcation and Chaos 13(10):2917-2934.

F. Dercole \& Y. Kuznetsov (2005). 'SlideCont: An Auto97 driver for bifurcation analysis of Filippov systems'. ACM Transactions on Mathematical Software 31:95-119.

M. di Bernardo, et al. (1999). 'Local Analysis of C-bifurcations in n-dimensional piecewise smooth dynamical systems'. Chaos, Solitons and Fractals 10:1881-1908.

E. J. Doedel, et al. (1998). AUTO97, Continuation and bifurcation software for ordinary differential equations.

V. Eyert (1996). 'A comparative study on methods for convergence acceleration of iterative vector sequences'. Journal of Computational Physics 124(0059):271-285.

U. Galvanetto \& S. Bishop (1999). 'Dynamics of a simple damped oscillator undergonig stick-slip vibrations'. Meccanica 34:337-347.

D. Gauthier, et al. (1994). 'Stabilizing unstable periodic orbits in a fast diode resonator using continuous time-delay autosynchronization'. Phys. Rev. E 50(3):2343-2346.

M. Hassouneh \& E. Abed (2004). 'Border collision bifurcation control of cardiac alternans'. Int. J. of Bifurcation and Chaos 14(9):3303-3315.

R. Horváth (2000). 'Experimental investigation of excited and self-excited vibration'. Master's thesis, University of Technology and Economics, Budapest, http://www.auburn. edu/ ${ }^{\text {horvaro/index } 2 . h t m l . ~}$

I. Kevrekidis, et al. (2004). 'Equation-free: The computer-aided analysis of complex multiscale systems'. AIChE Journal 50(11):1346-1355. 
Y. Kuznetsov (2004). Elements of Applied Bifurcation Theory. Springer Verlag. third edition.

Y. Kyrychko, et al. (2005). 'Real-time dynamic substructuring in a coupled oscillatorpendulum system'. Proc. Roy. Soc. London A 462:1271-1294.

K. Pyragas (1992). 'Continuous control of chaos by self-controlling feedback'. Phys. Lett. A 170:421-428.

K. Pyragas (2001). 'Control of chaos via an unstable delayed feedback controller'. Phys. Rev. Lett. 86(11):2265-2268.

J. Sieber \& B. Krauskopf (2006). 'Control-based continuation of periodic orbits with a time-delayed difference scheme'. Int. J. of Bifurcation and Chaos (in press). (http: //hdl.handle.net/1983/399).

C. Siettos, et al. (2004). 'Coarse Bifurcation Diagrams via Microscopic Simulators: a StateFeedback Control-Based Approach'. Int. J. of Bifurcation and Chaos 14(1):207-220.

G. Stépán \& T. Insperger (2004). 'Research on Delayed Dynamical Systems in Budapest'. Dynamical Systems Magazine http://www.dynamicalsystems.org/ma/ma/ display?item $=85$.

L. Trefethen (1996). 'Finite Difference and Spectral Methods for Ordinary and Partial Differential Equations'. Unpublished text, available at http://web.comlab.ox.ac.uk/ oucl/work/nick.trefethen/pdetext.html.

L. Trefethen \& M. Embree (2005). Spectra and Pseudospectra: The Behavior of Nonnormal Matrices and Operators. Princeton University Press. 